\title{
Long-Term Trends in Hospitalization and Outcomes in Adult Patients with Exacerbation of Chronic Obstructive Pulmonary Disease in Beijing, China, from 2008 to 2017
}

This article was published in the following Dove Press journal:

International Journal of Chronic Obstructive Pulmonary Disease

\section{Lirong Liang (D) \\ Changwei $\mathrm{Li}^{2}$ \\ Ye Shen ${ }^{2}$ \\ Hengmo Rong ${ }^{3}$ \\ Hang Jing ' \\ Zhaohui Tong ${ }^{3}$}

'Department of Clinical Epidemiology and Tobacco Dependence Treatment

Research, Beijing Institute of Respiratory

Medicine, Beijing Chaoyang Hospital, Capital Medical University, Beijing,

People's Republic of China; ${ }^{2}$ Department of Epidemiology and Biostatistics, University of Georgia College of Public Health, Athens, GA, USA; ${ }^{3}$ Department of Respiratory and Critical Care Medicine, Beijing Institute of Respiratory Medicine, Beijing Chaoyang Hospital, Capital Medical University, Beijing, People's Republic of China
Correspondence: Zhaohui Tong Department of Respiratory and Critical Care Medicine, Beijing Institute of Respiratory Medicine, Beijing Chaoyang Hospital, Capital Medical University, No. 8 Gongren Tiyuchang Nanlu, Chaoyang District, Beijing 100020, People's Republic of China

Tel +86-10-85231610

Fax +86-10-65060167

Email tongzhaohuicy@sina.com
Background and Objective: Chronic obstructive pulmonary disease (COPD) is a leading cause of morbidity and mortality worldwide. We aimed to evaluate the temporal trends in hospitalization and mortality from acute exacerbation of COPD (AECOPD) and the associated financial costs over a 10 -year period in Beijing, China.

Methods: Hospital admission records from 2008 to 2017 for all patients aged $\geq 20$ years with a primary discharge diagnosis of AECOPD were retrieved from the Beijing Public Health Information Center Database. Joinpoint regression was used to analyze trends and calculate the annual percentage change (APC) and average annual percent change (AAPC) for AECOPD hospitalization and mortality.

Results: A total of 337,802 AECOPD cases were recorded from 2008 to 2017. An inverse U-shaped trend in the AECOPD hospitalization rate was observed, showing an increase from 150.2 per 100,000 inhabitants in 2008 to 218.7 per 100,000 inhabitants in 2014 (APC: $5.5 \%$, 95\% CI: 2.9-8.2), before declining to 161.13 per 100,000 inhabitants in 2017 (APC: $-9.7 \%, 95 \%$ CI: -16.0 to-2.9). In-hospital mortality from AECOPD decreased significantly from $3.91 \%$ to $2.21 \%$ (AAPC: $-11.4 \%, 95 \% \mathrm{CI}:-15.5$ to -7.0 ). A decline in the median length of hospital stay from 13.0 days in 2008 to 12.0 days in $2017\left(P_{\text {trend }}<0.001\right)$ was accompanied by a decrease in the use of mechanical ventilation from 2012 to $2017\left(P_{\text {trend }}<0.001\right)$. However, the total hospitalization cost per case increased from 15953.5 yuan (USD \$2281.4) to 19874.5 yuan (\$2842.1) during the same period.

Conclusion: AECOPD remains a heavy burden on the health care system in Beijing. Strategies to better manage COPD and reduce hospitalizations from AECOPD are needed.

Keywords: COPD, exacerbation, hospitalization cost, mortality, outcome research

\section{Introduction}

Chronic obstructive pulmonary disease (COPD) is a leading cause of morbidity and mortality worldwide. ${ }^{1}$ In China, COPD was the third leading cause of death in 2013, accounting for more than 0.9 million deaths. ${ }^{2}$ Moreover, the prevalence of spirometry-defined COPD in China increased rapidly from $8.2 \%$ in $2002-2004$ to $13.7 \%$ in $2012-2015 .^{3,4}$ This increasing trend was especially strong among adults aged $\geq 40$ years. The high prevalence of smoking, heavy air pollution, and an aging population have contributed to the heavy burden of COPD in China. 
For patients with COPD, most morbidity and mortality result from acute exacerbations. Acute exacerbations of COPD (AECOPD) are pivotal events in the natural history of the disease and are associated with the disease's rapid progression and with impaired quality of life. AECOPD also accounts for most COPD-related health care costs. ${ }^{5,6}$ Several efforts, including the development of national guidelines to ensure proper care for patients with $\mathrm{COPD},{ }^{7,8}$ have been made to reduce hospitalizations for AECOPD in recent decades in China. Ambient air quality has also improved in recent years (2013-2017) in the country, resulting in substantial reductions in all-cause mortality ${ }^{9}$ and hospitalization for AECOPD. ${ }^{10}$ In 2015, many metropolitan cities in China adopted the Tobacco Control Regulations, ${ }^{11}$ but the effects of these efforts on long-term trends in AECOPD hospitalization and mortality remain unclear.

Therefore, the present study aimed to estimate the time trends in AECOPD hospitalization and mortality in Beijing, the capital of China, using city-wide representative hospital discharge data from 2008 to 2017. We also aimed to evaluate time trends in AECOPD-related parameters, including the use of ventilation, length of hospital stay (LOHS), and economic cost per patient.

\section{Materials and Methods}

\section{Data Source}

AECOPD hospitalization records were retrieved from a hospital discharge database operated by the Beijing Public Health Information Centre. In Beijing, only secondary- and tertiary-level hospitals can admit inpatients. These hospitals are required to submit the first page of a standardized medical record to the discharge database for each hospital discharge. This page includes data on patient demographic characteristics, hospital name, date of admission, primary discharge diagnosis and corresponding International Classification of Diseases, 10th Revision (ICD-10) code, and standard procedures. All hospitalization records for patients aged $\geq 20$ years with a primary discharge diagnosis of AECOPD (ICD-10 codes J44.0-J44.9) from 2008 to 2017 were included in the current analyses.

The present study was approved by the Research Ethics Board of Beijing Chaoyang Hospital (2018-ke-303). Data were de-identified before analysis. It is impossible to identify patients at the individual level either in this article or in the retrieved database. Given the anonymous and mandatory nature of the data, informed consent was not required or necessary.

\section{Statistical Analysis}

Descriptive statistics are presented as means \pm standard deviations or medians and interquartile ranges for continuous variables and as frequencies and percentages for categorical variables.

Annual hospitalization rates were calculated by dividing the total number of hospitalizations in a year for AECOPD by the corresponding number of inhabitants in the relevant population group according to the Beijing Municipal Bureau of Statistics. ${ }^{12}$ The in-hospital mortality (IHM) rate of AECOPD was also calculated for each year as the proportion of deaths among all hospitalized COPD patients. The age-standardized annual hospitalization rate and the IHM rate for each year were estimated using the direct method, based on the 2010 population census. ${ }^{13} \mathrm{We}$ also examined the trends in hospitalization and IHM by sex, age group (20-39, 40-59, 60-79, and $\geq 80$ years) and hospital type (secondary and tertiary).

Use of mechanical ventilation (MV), including noninvasive MV or invasive MV, was determined using ICD-10 code J15.501, which was available in the dataset from 2012 to 2017. LOHS and total hospitalization costs were also estimated for each year. Annual hospitalization costs were adjusted for the inflation rate in China with reference to 2017. The annual inflation rate was obtained from the National Bureau of Statistics of China. ${ }^{14}$

The time trend analysis was conducted using Poisson regression models for hospitalization rates and logistic regression models for IHM and MV, with calendar year as the timescale. Time trends for LOHS and total costs were tested using the Kruskal-Wallis test and simple linear regression analysis, respectively. All statistical analyses were performed using SAS 9.4 (Cary, NC, USA). Statistical significance was set as two-tailed $P<0.05$.

To identify turning points in the time trends and to estimate the annual percentage change (APC) for ageadjusted hospitalization and IHM rates, the United States National Cancer Institute's Joinpoint Regression Program was used for each time segment. The program assumes that rates changed at a constant percentage every year on a log scale in each time segment. The average annual percent change (AAPC) - a weighted average of APCs from the Joinpoint models, with weights equal to the length of the APC interval-was also computed as a summary measure of the trend over the whole observation period. ${ }^{15}$ A two-tailed $P$ value $<0.05$ was considered statistically significant. 


\section{Results}

\section{AECOPD Hospitalization Rates 2008-2017}

We identified 337,802 patient discharge records $(63.5 \%$ male, $\mathrm{n}=213,037)$, submitted by 78 tertiary hospitals and 77 secondary hospitals, with a primary diagnosis of AECOPD from 2008 to 2017 in Beijing, China. Most patients $(69.7 \%)$ were admitted to tertiary hospitals. As shown in Table 1 (Figure 1), the overall hospitalization rate increased from 150.15 per 100,000 inhabitants in 2008 to 218.74 per 100,000 in 2014 and then declined to 161.13 per 100,000 in 2017. The hospitalization rate was higher for men than for women and increased with age, with the highest rate seen among patients aged $\geq 80$ years $(P<0.001)$ in each calendar year. Tertiary hospitals had higher hospitalization rates than did secondary hospitals.

\section{In-Hospital Mortality of Hospitalized Patients with AECOPD 2008-20I7}

Table 2 (Figure 2) presents IHM for each calendar year. During the study period, the overall IHM among patients with AECOPD decreased significantly from $3.91 \%$ to $2.21 \%$ ( $\left.P_{\text {trend }}<0.001\right)$. IHM increased with age $(P<0.001)$ and was higher for women than for men $(P<0.001)$ in each calendar year. IHM was higher in tertiary hospitals than in secondary hospitals in all years except 2008 and 2014.

\section{Trends in Age-Adjusted AECOPD} Hospitalization and IHM Rates 2008-20I7

As shown in Table 3, age-adjusted hospitalization for AECOPD increased by $5.5 \%$ (95\% confidence interval [CI]: $2.9-8.2 \%, P=0.003)$ per year from 2008 to 2014 (APC: $5.5 \%, 95 \%$ CI: 2.9-8.2\%, $P=0.003$ ) (Table 3) and then decreased by $9.7 \%$ (95\% CI: $2.9-16.0 \%, P=0.015)$ per year from 2014 to 2017 . The trends in the hospitalization rate differed by sex (Table 3). For men, the rate increased significantly from 2008 to 2014 (APC: $8.0 \%$, 95\% CI: 4.9\%$11.1 \%, P=0.001)$ and then decreased slightly from 2014 to 2017 (APC: $-6.5 \%$, 95\% CI: $-13.5 \%-1.1 \%, P=0.078$ ), which led to an overall increasing trend (AAPC: $2.9 \%$, 95\% CI: $0.4 \%-5.5 \%, P=0.022$ ). For women, the rate peaked early in 2013 and then decreased significantly from 2013 to 2017 (APC: $-12.3 \%$, 95\% CI: $-20.3 \%-3.4 \%, P=0.017$ ), leading to an overall decreasing but non-significant change (AAPC: $-3.6 \%, 95 \% \mathrm{CI}:-7.7 \%-0.7 \%, P=0.099$ ).

Trends in hospitalization rates also varied by age group. A significant trend was observed only among those aged 20-39 years (AAPC: $2.6 \%$, 95\% CI: $0.4 \%-$ $4.8 \%, P=0.024)$. The other three age groups (40-59, 60-79, and $\geq 80$ years) had inverse U-shaped trends, which peaked in 2014 for those aged 40-59 and 60-79 years and in 2013 for the oldest age group. From 2008 to 2014, hospitalization rates increased significantly for those aged 40-59 years (APC: 11.5\%, 95\% CI: 5.8\%-17.5\%,

Table I Hospitalization Rates ${ }^{\mathrm{a}}$ Among Patients Hospitalized for Acute Exacerbations of Chronic Obstructive Pulmonary Disease, 2008-2017

\begin{tabular}{|c|c|c|c|c|c|c|c|c|c|c|c|}
\hline & 2008 & 2009 & 2010 & 2011 & 2012 & 2013 & 2014 & 2015 & 2016 & 2017 & $P$ \\
\hline Number & 23194 & 25341 & 29492 & 29870 & 36539 & 40315 & 45681 & 34491 & 37403 & 35476 & $0.227^{\mathrm{b}}$ \\
\hline In 100,000 habitants & 150.15 & 155.09 & 172.40 & 165.02 & 191.55 & 200.87 & 218.74 & 159.03 & 167.59 & 161.13 & \\
\hline Gender & & & & & & & & & & & $<0.001^{c}$ \\
\hline Women & 131.86 & 133.97 & 147.40 & 139.78 & 155.18 & 156.47 & 153.52 & 106.39 & 106.19 & 98.91 & \\
\hline Men & 166.90 & 174.30 & 195.19 & $187.9 \mid$ & 224.74 & 241.67 & 279.40 & 208.02 & 225.04 & 221.23 & \\
\hline Age, Years & & & & & & & & & & & $<0.001^{c}$ \\
\hline $20-39$ & 8.92 & 7.43 & 8.04 & 8.46 & 9.31 & 10.00 & 8.94 & 8.64 & 10.66 & 10.15 & \\
\hline $40-59$ & 49.59 & 50.71 & 52.47 & 52.32 & 65.87 & 78.31 & 94.39 & 64.67 & 65.91 & 58.68 & \\
\hline $60-79$ & 687.99 & 726.66 & 790.13 & 741.65 & 860.85 & 886.51 & 975.33 & 725.30 & 770.75 & 726.35 & \\
\hline$\geq 80$ & 2252.33 & 2273.00 & 2734.15 & 2659.42 & 3002.08 & 3079.30 & 3161.54 & 2198.06 & 2269.74 & 2381.07 & \\
\hline Hospital Level & & & & & & & & & & & $<0.001^{c}$ \\
\hline Secondary hospitals & 45.03 & 45.39 & 51.60 & 50.23 & 59.52 & 60.07 & 67.18 & 47.18 & 49.80 & 46.60 & \\
\hline Tertiary hospitals & 105.11 & 109.70 & $|20.8|$ & 114.79 & 132.03 & 140.80 & 151.57 & 111.84 & 117.78 & 114.53 & \\
\hline
\end{tabular}

Notes: ${ }^{a}$ Hospitalization rates were calculated by dividing the number of cases per year for the relevant sex/age group by the corresponding number of persons in that population group, age-adjusted based on the 2010 population census. ${ }^{b} P$ value for time trend of hospitalization rates estimated using Poisson regression models. ${ }^{c} P$ value for subgroup comparisons estimated using Poisson regression models, with adjustment for year. 
A

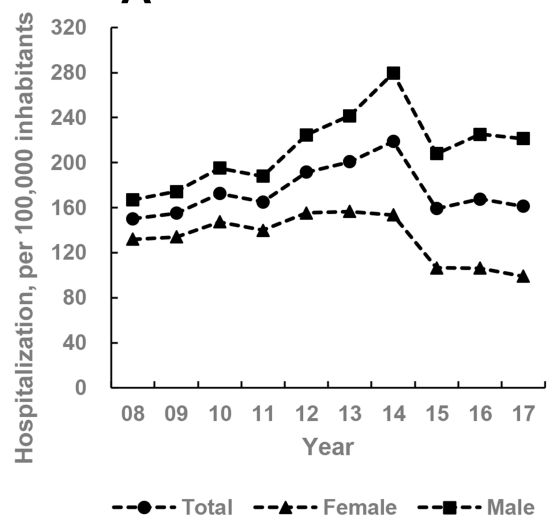

B

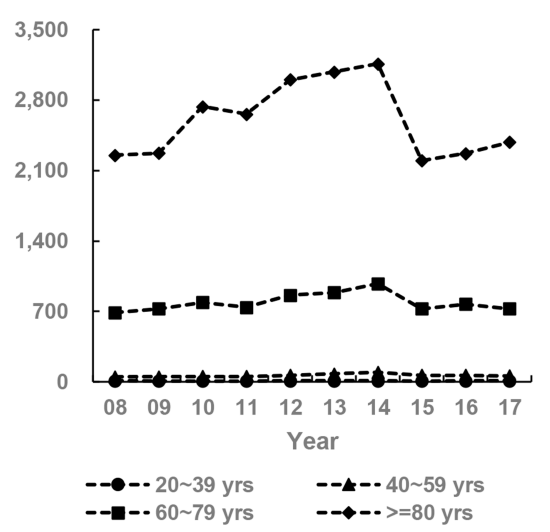

C

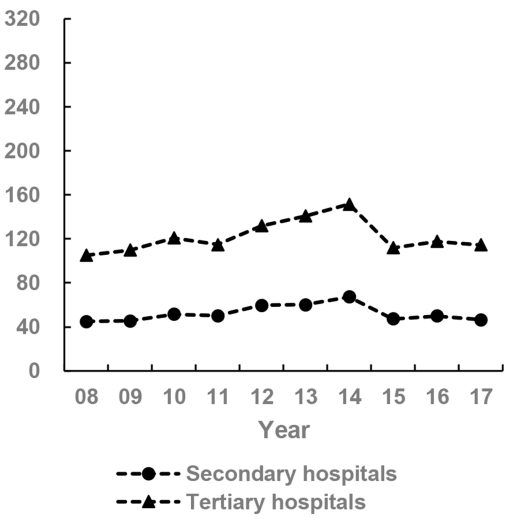

Figure I Hospitalization rates among patients hospitalized with acute exacerbations of chronic obstructive pulmonary disease (AECOPD) by gender, age, and hospital type from 2008 to 2017 in Beijing, China. Data presented as annual hospitalization rates calculated by dividing the total number of hospitalizations for AECOPD in a year by the corresponding number of inhabitants in that population group according to the Beijing Municipal Bureau of Statistics. (A) Overall and gender-specific hospitalization rates. (B) Age-specific hospitalization rates. (C) Hospital type-specific hospitalization rates.

$P=0.003$ ) and 60-79 years (APC: $4.8 \%, 95 \%$ CI: $1.9 \%-$ $7.8 \%, P=0.007)$. From 2014 to 2017 , the rate decreased significantly for those aged 60-79 years (APC: $-8.8 \%$, 95\% CI: $-15.8 \%-1.1 \%, P=0.033$ ) and nominally for those aged 40-59 years (APC: $-12.7 \%$, 95\% CI: $-24.9 \%-1.5 \%, P=0.068)$. However, for those aged $\geq 80$ years, the inverse U-shaped trend was not significant overall or for any time segment. The magnitude of change was largest for those aged 40-59 years.

Inverse U-shaped trends in hospitalization rates were observed for both secondary and tertiary hospitals.
However, from 2008 to 2014, a faster increase was observed in secondary hospitals (APC: 6.0\%, 95\% CI: $3.0-9.2 \%$ ) than in tertiary hospitals (APC: $5.3 \%, 95 \%$ CI: $2.7-7.9 \%$ ), and a faster decrease was seen in secondary hospitals (APC: $-11.3 \%, 95 \% \mathrm{CI}:-18.5 \%-3.6 \%$ ) than in tertiary hospitals (APC: $-8.9 \%, 95 \% \mathrm{CI}: 15.3 \%-2.1 \%$ ) from 2014 to 2017.

In contrast to the findings for hospitalization rates, IHM from AECOPD decreased significantly throughout the study period (AAPC: $-11.4 \%$, 95\% CI: $-15.5--7.0$, $P<0.001)$. Among women, IHM decreased slowly from

Table 2 In-Hospital Mortality ${ }^{a}$ Among Patients Hospitalized for Acute Exacerbations of Chronic Obstructive Pulmonary Disease, 2008-2017

\begin{tabular}{|c|c|c|c|c|c|c|c|c|c|c|c|}
\hline & 2008 & 2009 & 2010 & 2011 & 2012 & 2013 & 2014 & 2015 & 2016 & 2017 & $P$ \\
\hline Number & 907 & 884 & 1147 & 962 & 1150 & 1252 & 1316 & 832 & 899 & 785 & $<0.001^{b}$ \\
\hline IHM & 3.91 & 3.49 & 3.89 & 3.22 & 3.15 & 3.11 & 2.88 & 2.41 & 2.40 & 2.21 & \\
\hline Gender & & & & & & & & & & & $<0.001^{c}$ \\
\hline Women & 3.80 & 3.71 & 4.06 & 3.17 & 3.10 & 3.36 & 3.36 & 2.87 & 2.73 & 2.59 & \\
\hline Men & 3.99 & 3.33 & 3.77 & 3.26 & 3.18 & 2.95 & 2.63 & 2.18 & 2.25 & 2.04 & \\
\hline Age, Years & & & & & & & & & & & $<0.001^{c}$ \\
\hline $20 \sim 39$ & 0.00 & 0.00 & 0.00 & 0.29 & 0.00 & 0.00 & 0.00 & 0.00 & 0.00 & 0.00 & \\
\hline $40 \sim 59$ & 0.97 & 0.73 & 1.13 & 0.87 & 0.49 & 0.63 & 0.29 & 0.49 & 0.34 & 0.28 & \\
\hline $60 \sim 79$ & 2.93 & 2.47 & 2.87 & 2.01 & 2.02 & 2.06 & 1.72 & 1.37 & 1.25 & 1.13 & \\
\hline$\geq 80$ & 6.97 & 6.40 & 6.38 & 5.66 & 5.49 & 5.19 & 5.17 & 4.33 & 4.45 & 4.07 & \\
\hline Hospital Level & & & & & & & & & & & $<0.001^{c}$ \\
\hline Secondary hospitals & 4.06 & 2.92 & 3.67 & 2.76 & 2.95 & 2.90 & 2.92 & 2.45 & 2.15 & 2.10 & \\
\hline Tertiary hospitals & 3.85 & 3.72 & 3.98 & 3.42 & 3.24 & 3.20 & 2.86 & 2.39 & 2.51 & 2.26 & \\
\hline
\end{tabular}

Notes: aln-hospital mortality was calculated by dividing the number of deaths among hospitalized subjects in the relevant sex/age group by the corresponding number of persons in that population group according to the Bureau of Statistics of China's 2010 data. ${ }^{b} P$ value for time trend of IHM estimated using logistic regression models. ${ }^{c} P$ value for subgroup comparisons estimated using logistic regression models, with adjustment for year. 

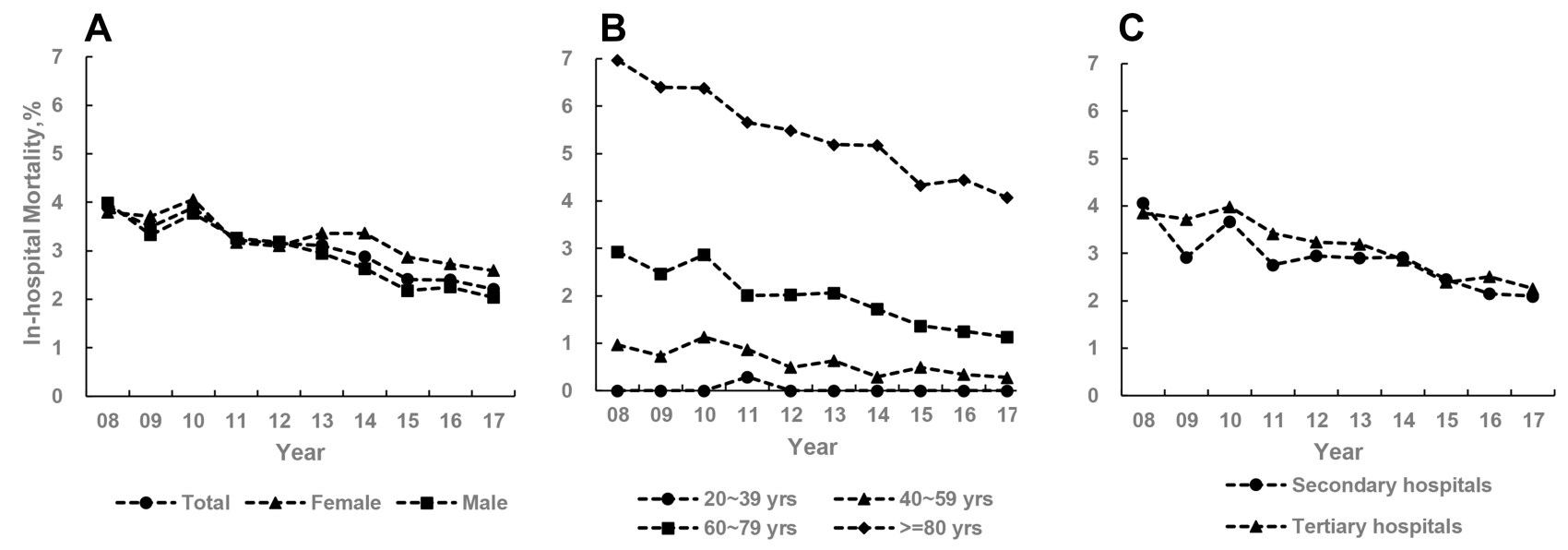

Figure 2 In-hospital mortality among patients hospitalized acute exacerbations of chronic obstructive pulmonary disease from 2008 to 2017 in Beijing, China. (A) Overall and gender-specific in-hospital mortality. (B) Age-specific in-hospital mortality. (C) Hospital type-specific in-hospital mortality.

2008 to 2014 (APC: $-7.0 \%, P=0.002$ ) and then decreased rapidly from 2014 to 2017 (APC: $-24.0 \%, P=0.006$ ). The largest IHM decrease was observed among those aged 20-59 years (APC: $-13.2 \%, P=0.012$ ) followed by those aged 60-79 years (APC: $-9.4 \%, P<0.001$ ) and then those aged $\geq 80$ years (APC: $-6.2 \%, P<0.001$ ). Interestingly, changes in IHM were similar for secondary and tertiary hospitals (APC: $-11.4 \%$ vs $-11.1 \%$, respectively).

\section{Trends in Mechanical Ventilation, LOHS, and Costs, 2008-2017}

MV use rapidly increased, from $16.3 \%$ in 2012 to $22.1 \%$ in 2014 , and then decreased to $11.97 \%$ in 2017 . During the same period, average LOHS dropped from 16.7 days to 13.2 days $\left(P_{\text {trend }}<0.001\right)$, and the median cost of each AECOPD hospitalization dramatically increased, from 10875.1 yuan (USD \$1555.1) in 2008 to 14,698.4 yuan (\$2101.9) in 2017, after correcting for inflation $\left(P_{\text {trend }}<0.001\right.$, Table 4$)$.

\section{Discussion}

In this representative sample of hospitalization records of patients with AECOPD in Beijing, China, from 2008 to 2017, we identified a marked shift in the rate of hospitalization for AECOPD, with an increasing trend from 2008 to 2014 followed by a decreasing trend. In contrast, IHM from AECOPD decreased significantly over the 10-year period, and this shift was accompanied by an increasing average cost per case. Interestingly, the use of MV and LOHS decreased over time.
The increasing rates of hospitalization for AECOPD from 2008 to 2014 might be caused by increased prevalence rates of COPD and smoking and by population aging. ${ }^{4,16}$ Improvements in economic status and health insurance may also partly contribute to the increasing burden. ${ }^{17-19}$ The downward trend in hospitalization rates since 2014 might be linked to the improved management of stable COPD and improvements in the treatment of AECOPD. The implementation of several public policies might also be attributable to the decreasing hospitalization rates during this period. In 2013, the Air Pollution Prevention and Control Action Plan (APPCAP) was launched by the Chinese Government to curb air pollution and reach target concentrations by 2017. ${ }^{9}$ Further, a previous study has reported a significant association between improvements in overall air quality and the declining rates of hospitalization for AECOPD from 2013 to 2017 in Beijing. ${ }^{10}$ In addition, the "Beijing Tobacco Control Regulation" was adopted in 2015, and the prevalence of cigarette smoking then declined in 2016 in Beijing (from $23.4 \%$ in 2015 to $22.3 \%$ in 2016). ${ }^{11}$ The long-term effect of these policies on COPD warrants continuous documentation and further investigation.

In the current study, men had higher AECOPD hospitalization rates than did women, which is in line with previously reported findings in Spain $^{20}$ but not in the United States. $^{21}$ The substantially higher hospitalization rate in women compared to men might mainly due to the significantly higher prevalence of COPD in men $(11.9 \%)$ than those in women $(5.4 \%){ }^{4}$ We also revealed genderspecific time trends in hospitalization rates. For men, the hospitalization rate increased over the 10-year study 


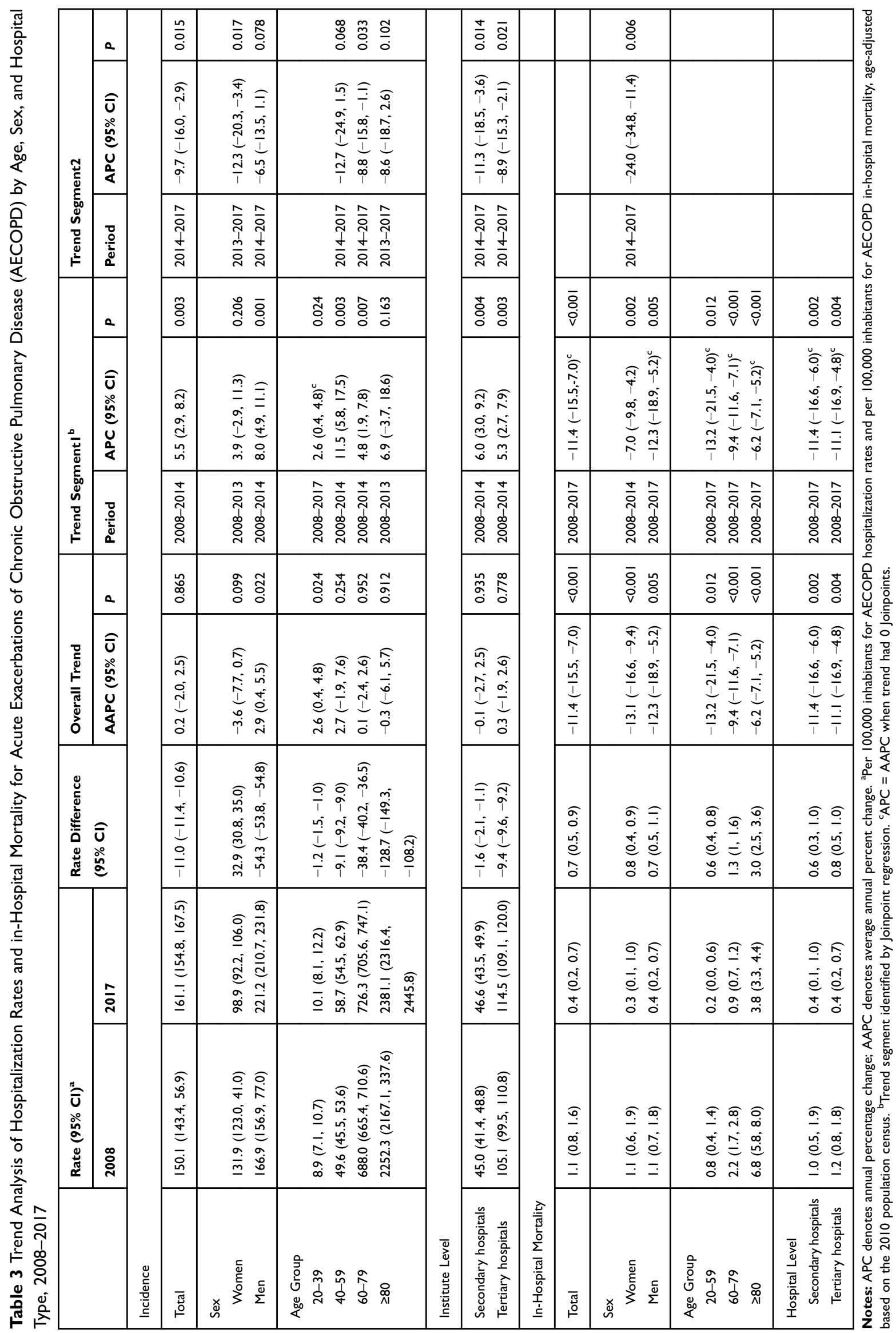


Table 4 Use of Mechanical Ventilation, Length of Hospital Stay (LOHS), and Costs for Acute Exacerbations of Chronic Obstructive Pulmonary Disease, 2008-2017

\begin{tabular}{|c|c|c|c|c|c|c|c|c|c|c|c|}
\hline & 2008 & 2009 & 2010 & 2011 & 2012 & 2013 & 2014 & 2015 & 2016 & 2017 & $P$ \\
\hline $\begin{array}{l}\text { Mechanical Ventilation } \\
\mathrm{N}, \%\end{array}$ & - & - & - & - & $\begin{array}{l}2031 \\
(16.33 \%)\end{array}$ & $\begin{array}{l}2588 \\
(20.81 \%)\end{array}$ & $\begin{array}{l}2746 \\
(22.08 \%)\end{array}$ & $\begin{array}{l}1663 \\
(13.37 \%)\end{array}$ & $\begin{array}{l}1825 \\
(14.68 \%)\end{array}$ & $\begin{array}{l}1489 \\
(11.97 \%)\end{array}$ & $<0.001^{a}$ \\
\hline $\begin{array}{l}\text { Average hours of receiving } \\
\text { mechanical ventilation, } \\
\text { hours } \\
\text { Mean } \\
\text { SD } \\
\text { Median } \\
\text { QI-Q3 }\end{array}$ & $\bar{r}$ & $\bar{r}$ & $\bar{r}$ & $\bar{r}$ & $\begin{array}{l}255.23 \\
435.35 \\
130 \\
50-300\end{array}$ & $\begin{array}{l}237.65 \\
443.97 \\
138 \\
55-277\end{array}$ & $\begin{array}{l}228.47 \\
333.56 \\
137 \\
67-282\end{array}$ & $\begin{array}{l}244.95 \\
309.20 \\
147 \\
64-311\end{array}$ & $\begin{array}{l}220.83 \\
273.13 \\
140 \\
48-287\end{array}$ & $\begin{array}{l}233.57 \\
273.96 \\
139 \\
57-296\end{array}$ & $0.118^{b}$ \\
\hline $\begin{array}{l}\text { LOHS, Days } \\
\text { Mean } \\
\text { SD } \\
\text { Median } \\
\text { QI } \\
\text { Q3 }\end{array}$ & $\begin{array}{l}16.71 \\
21.80 \\
13 \\
9 \\
19\end{array}$ & $\begin{array}{l}16.22 \\
23.60 \\
13 \\
9 \\
18\end{array}$ & $\begin{array}{l}15.75 \\
15.61 \\
13 \\
9 \\
18\end{array}$ & $\begin{array}{l}15.42 \\
22.93 \\
12 \\
8 \\
17\end{array}$ & $\begin{array}{l}14.44 \\
13.30 \\
12 \\
8 \\
17\end{array}$ & $\begin{array}{l}14.05 \\
13.03 \\
12 \\
8 \\
17\end{array}$ & $\begin{array}{l}14.18 \\
11.33 \\
12 \\
8 \\
17\end{array}$ & $\begin{array}{l}13.91 \\
9.84 \\
12 \\
8 \\
17\end{array}$ & $\begin{array}{l}13.50 \\
8.62 \\
12 \\
8 \\
16\end{array}$ & $\begin{array}{l}13.18 \\
8.04 \\
12 \\
8 \\
16\end{array}$ & $<0.001^{b}$ \\
\hline $\begin{array}{l}\text { Cost }^{\mathrm{d}}, \mathrm{CNY} \\
\text { Mean } \\
\text { SD } \\
\text { Median } \\
\text { QI } \\
\text { Q3 }\end{array}$ & $\begin{array}{l}15953.5 \\
22542.2 \\
10875.1 \\
6378.3 \\
18064.8\end{array}$ & $\begin{array}{l}18483 \\
3050|.| \\
12 \mid 20.9 \\
7079.5 \\
20493.5\end{array}$ & $\begin{array}{l}19982.9 \\
398 \mid 2.5 \\
13021.6 \\
7815.3 \\
21494.1\end{array}$ & $\begin{array}{l}19205.1 \\
33546.2 \\
12747.1 \\
7989.9 \\
20229.4\end{array}$ & $\begin{array}{l}18988 \\
29203.9 \\
13035.4 \\
8262.8 \\
20572.1\end{array}$ & $\begin{array}{l}19163 \\
27404.4 \\
13544.9 \\
8940.5 \\
21199.3\end{array}$ & $\begin{array}{l}19482 \\
26977.3 \\
13718.6 \\
9366.5 \\
21137.8\end{array}$ & $\begin{array}{l}200 \mid 2.5 \\
24 \mid 36.5 \\
146 \mid 5.1 \\
9809.6 \\
22238\end{array}$ & $\begin{array}{l}20220.8 \\
22693.4 \\
14777.7 \\
10194.2 \\
23044.1\end{array}$ & $\begin{array}{l}19874.5 \\
21485.9 \\
14698.4 \\
10282.3 \\
22470.3\end{array}$ & $<0.001^{\mathrm{c}}$ \\
\hline
\end{tabular}

Notes: — indicates that data were unavailable. ${ }^{a} P$ value for time trend estimated using logistic regression models. ${ }^{b} P$ value comparing medians over the study period calculated using the Kruskal-Wallis test. ${ }^{c} P$ value for time trend estimated using simple linear regression analysis. ${ }^{\mathrm{d}}$ All costs shown are adjusted for the increment of inflation in 2017.

period, whereas, for women, the hospitalization rate decreased from 2013 to 2017 . The upward trend in hospitalization rates for men may be caused by men's higher prevalence of smoking and COPD. ${ }^{4,16}$ The downward trend observed for women may reflect women's higher levels of awareness and treatment and better compliance with disease management. ${ }^{22}$ We also found an inverse U-shaped trend in hospitalization rates for all age groups except those aged 20-39 years, whose rate continued to increase from 2008 to 2017. This increasing rate may be partly explained by an increased prevalence of $\mathrm{COPD}^{4}$ and early detection of COPD in this age group.

Compared with secondary hospitals, in tertiary hospitals, the rate of hospitalization for AECOPD was higher over the study period and decreased more slowly from 2014 to 2017 . The difference by hospital type was likely driven by the fact that patients in China prefer higher-level hospitals and can access the system at any level because there are no formal gatekeepers in the hospital care system..$^{23}$ In 2013, regional medical alliances were established by all hospitals in each district of Beijing in an effort to deliver hierarchical care to patients. ${ }^{18}$ These alliances aim for primary and secondary hospitals to have more patients with less severe conditions and for tertiary hospitals to focus on more serious and complicated cases and treat fewer patients with common conditions. However, our results did not reveal improvements in terms of overcrowding in tertiary hospitals or the underutilization of lower-hospitals for AECOPD 4 years after the establishment of the regional medical alliances.

IHM from AECOPD declined over the study period in Beijing. Similar declining trends have been reported in previous studies using hospital discharge databases in other countries. However, IHM from AECOPD was higher in other countries than in Beijing. ${ }^{21,24,25}$ Beijing's success in terms of IHM may be explained by a number of factors, including increased medical care quality and effectiveness of treatment for AECOPD. It is also possible that the severity of AECOPD declined, resulting in better patient survival rates. Improvements in air quality in Beijing in the past 5 years may also have contributed to the decreasing IHM. ${ }^{9}$

Consistent with previous studies, we found that IHM increased with age in all calendar years and was higher for 
women in all years except 2008, 2011, and 2012. ${ }^{20,21}$ From 2014 to 2017, an especially strong declining trend was observed among those aged 20-59 years and a more dramatic decrease was seen among women. These downward trends in IHM may indicate that younger and female patients with severe AECOPD are more likely to benefit from improved inpatient care. The similarity in declining trends found in secondary and tertiary hospitals indicates equal improvements over time in AECOPD treatment in both types of facilities. In addition, it was found higher IHM in women than those in men during the study period. In this largely descriptive analysis, we were unable to identify the sources which explained the differences. Future research is needed to elucidate the determinants of gender differences.

The downward trend in IHM was accompanied by declining use of MV, shorter LOHS, and higher hospitalization costs. Similar trends have previously been reported in other countries. ${ }^{20,26}$ Researchers have demonstrated that noninvasive $\mathrm{MV}$ can reduce the need for invasive $\mathrm{MV}$, decrease LOHS, and improve IHM, leading to recommendations for noninvasive MV as a first-line ventilation modality for acute respiratory failure caused by AECOPD. ${ }^{27,28}$ We could not assess differences between noninvasive ventilation and invasive MV due to lack of related information. Noticeably, the rates of receiving MV were lower in our research than those reported in the United States (from $<15.0 \%$ in 2001 to about $20.0 \%$ in 2011), ${ }^{26}$ but higher than those in Spain (from $1.4 \%$ in 2006 to $2.9 \%$ in 2010). ${ }^{20}$ Higher rates of MV might reflect that the enrolled patients were more severe. However, it was observed that relatively lower IHM in our present study $(2.1 \%$ in 2017$)$ than those in the other two studies $(3.9 \%$ in 2010 in the United States and 4.5\% in 2010 in Spain, respectively). ${ }^{20,26}$ Because of cultural perception or affordability, certain dying or terminal patients in China might withdraw from hospitalized treatment, which could underestimate the IHM rates.

Median hospitalization costs increased during the study period, despite a decrease in LOHS, even after adjusting for inflation, which may point to increases in the availability and utilization of diagnostic and treatment modalities, leading to rising health care costs in Beijing over the past decade. The overall cost of care at the patient level has also been shown to be associated with COPD severity and number of comorbidities. ${ }^{29,30}$ Unfortunately, our database did not allow us to stratify patients with AECOPD by severity or number of comorbidities at admission.
A similar city-wide study conducted by Liu et al in Dalian, China, reported the temporary trends of patients with COPD from 2005 to 2015, showing the increase in the hospitalization rate. ${ }^{31}$ With regard to their enrolled cases with both primary diagnosis and up to 5 secondary diagnoses of COPD (ICD codes J40-J44.9, J47.0, J47.1, and J47.9), the findings reported by Liu et al were not directly comparable to our results which focus on the cases with primary diagnosis of AECOPD (ICD-10 codes J44.0J44.9). However, Liu et al were also found similar trends to our findings, with a decline in the median LOS and an increase in the total hospitalization cost per case. ${ }^{31}$ Altogether, these findings demonstrate that COPD remains a heavy burden on the health care system in China. Because hospitalizations for AECOPD are responsible for most of the expenses related to the disease, ${ }^{5,30}$ strategies to prevent admission/readmission among patients with COPD are crucial for reducing the economic burden of COPD.

A major strength of our study was that we used representative data to examine 10-year trends in hospitalization and outcomes for AECOPD among adults aged 20 years and older in Beijing. Moreover, we used the Joinpoint Regression Program to identify points where these trends changed and to estimate the APC for the purpose of exploring underlying reasons, such as changes in environmental risk factors and the implementation of specific policies or programs at certain time points. However, our study also had several limitations. First, like all studies using data from medical records, our analyses were limited to the variables that were available in the database. Although hospital readmissions for AECOPD contribute to the increasing costs of COPD, ${ }^{5,30}$ we could not determine whether a hospitalization record was for a patient experiencing their first episode of exacerbation or recurrent exacerbations, because the database contained no unique subject identifier. We were therefore also unable to assess the AECOPD readmission rate. Second, the present time trend analyses of disease rates were ecological descriptive analyses, which are inevitably subject to the ecological fallacy. Therefore, all etiological interpretations require further confirmation by analytic epidemiological studies. Third, it is possible that the younger cases hospitalization for AECOPD in the 20-39 years age-subgroup had alternative diagnoses, which might lead to misclassification bias. Given the relatively low proportion of this age subgroup in the total population $(3476 / 337,802,1.03 \%)$, this bias had less impact on the main findings. Finally, the data are from Beijing, and our findings may not be generalizable to patients with AECOPD in other provinces of China. Future studies 
using nationwide records are warranted to better reflect the burden of this condition in China as a whole.

\section{Conclusions}

This study provides clear and valid data indicating a distinct shift in hospital admission rates for AECOPD in Beijing, China, from 2008 to 2017. In addition, concomitant reductions in IHM and LOHS for AECOPD and increasing hospitalization costs were identified. Future strategies to reduce the burden of hospitalizations for COPD are needed.

\section{Abbreviations}

COPD, chronic obstructive pulmonary disease; AECOPD, acute exacerbation of COPD; APC, annual percentage change; AAPC, average annual percent change; $\mathrm{CI}$, confidence interval; LOHS, length of hospital stay; ICD-10, International Classification of Diseases, 10th Revision; MV, mechanical ventilation.

\section{Ethics Approval and Informed Consent}

This study was approved by the Research Ethics Board of Beijing Chaoyang Hospital (2018-ke-303). Data were deidentified before analysis. Given the anonymous and mandatory nature of the data, informed consent was not required or necessary.

\section{Acknowledgment}

We thank the data collection teams.

\section{Author Contributions}

LLR conceived and designed the study. LLR performed the statistical analyses and drafted the manuscript. CWL and ZHT supervised the data analysis and edited the manuscript. All authors contributed to data analysis, drafting or revising the article, gave final approval of the version to be published, and agree to be accountable for all aspects of the work.

\section{Disclosure}

The authors declare that they have no competing interests in this work.

\section{References}

1. GBD 2016 Causes of Death Collaborators. Global, regional, and national age-sex-specific mortality for 282 causes of death in 195 countries and territories, 1980-2017: a systematic analysis for the Global Burden of Disease Study 2017. Lancet. 2018;392:1736-1788.
2. Zhou M, Wang H, Zhu J, et al. Cause-specific mortality for 240 causes in China during 1990-2013: a systematic subnational analysis for the Global Burden of Disease Study 2013. Lancet. 2016;387:251-272. doi:10.1016/S0140-6736(15)00551-6

3. Zhong N, Wang C, Yao W, et al. Prevalence of Chronic Obstructive Pulmonary Disease in China. Am J Respir Crit Care Med. 2007;176 (8):753-760. doi:10.1164/rccm.200612-1749OC

4. Wang C, Xu J, Yang L, et al. Prevalence and risk factors of chronic obstructive pulmonary disease in China (the China Pulmonary Health [CPH] study): a national cross-sectional study. Lancet (London, England). 2018;391(10131):1706-1717. doi:10.1016/S01406736(18)30841-9

5. Khakban A, Sin DD, FitzGerald JM, et al. Ten-year trends in direct costs of COPD: a population-based study. Chest. 2015;148 (3):640-646. doi:10.1378/chest.15-0721

6. Zhu B, Wang Y, Ming J, et al. Disease burden of COPD in China: a systematic review. Int $J$ Chron Obstruct Pulmon Dis. 2018;13:1353-1364. doi:10.2147/COPD.S161555

7. COPD Group of Chinese Society of Respiratory Disease. Guideline for diagnosis and treatment of chronic obstructive pulmonary disease (revised edition in 2013). Chin J Front Med Sci. 2013;6:67-70.

8. Chinese Expert Group on the diagnosis and treatment of acute exacerbation of chronic obstructive pulmonary disease (AECOPD). Chinese expert consensus on the diagnosis and treatment of acute exacerbation of chronic obstructive pulmonary disease (AECOPD) (revised edition in 2014). Int J Respir. 2014;34:1-11.

9. Huang J, Pan X, Guo X, et al. Health impact of China's air pollution prevention and control action plan: an analysis of national air quality monitoring and mortality data. Lancet Planet Health. 2018;2:e313e23. doi:10.1016/S2542-5196(18)30141-4

10. Liang L, Cai Y, Barratt B, et al. Associations between daily air quality and hospitalisations for acute exacerbation of chronic obstructive pulmonary disease in Beijing, 2013-17: an ecological analysis. Lancet Planet Health. 2019;3:e270-e9. doi:10.1016/S2542-5196(19) 30085-3

11. Li YQ, Shi JH, Cao Y, et al. [One year after the implementation of 2015 tobacco control regulation on persons aged 15 years and over tobacco use in Beijing]. Zhonghua Liu Xing Bing Xue Za Zhi. 2018;39:1188-1192. Chinese. doi:10.3760/cma.j.issn.0254-6450.2018.09.009

12. Beijing Municipal Bureau of Statistics. http://www.bjstats.gov.cn. Accessed October 18, 2018.

13. National Bureau of Statistics of China. 2010 Population census. Available from: http://www.stats.gov.cn/english/Statisticaldata/ CensusData/. Accessed October 28, 2018.

14. National Bureau of Statistics of China. Available from: http://data. stats.gov.cn/easyquery.htm?cn=C01. Accessed November 9, 2018.

15. HJ K, Fay MP, Feuer EJ, Midthune DN. Permutation tests for joinpoint regression with applications to cancer rates. Stat Med. 2000;19:335-351. doi:10.1002/(SICI)1097-0258(20000215)19:3<335:: AID-SIM336>3.0.CO;2-Z

16. Chinese Center for Disease Control and Prevention. 2015 Chinese Adult Tobacco Survey Country Reports; 2015.

17. Meng Q, Xu L, Zhang Y, et al. Trends in access to health services and financial protection in China between 2003 and 2011: a cross-sectional study. Lancet. 2012;379:805-814. doi:10.1016/ S0140-6736(12)60278-5

18. Li L, Fu H. China's health care system reform: progress and prospects. Int J Health Plann Manage. 2017;32:240-253. doi:10.1002/ hpm. 2424

19. Pan J, Lei X, Liu GG. Health insurance and health status: exploring the causal effect from a policy intervention. Health Econ. 2016;25:1389-1402. doi:10.1002/hec.3225

20. de Miguel-diez J, Jimenez-Garcia R, Hernandez-Barrera V, et al. Trends in hospital admissions for acute exacerbation of COPD in Spain from 2006 to 2010. Respir Med. 2013;107:717-723. doi:10.1016/j.rmed.2013.01.007 
21. Jinjuvadia C, Jinjuvadia R, Mandapakala C, et al. Trends in outcomes, financial burden, and mortality for acute exacerbation of chronic obstructive pulmonary disease (COPD) in the United States from 2002 to 2010. Copd. 2017;14:72-79. doi:10.1080/15412555.2016.1199669

22. Kurmi OP, Davis KJ, Hubert Lam KB, et al. Patterns and management of chronic obstructive pulmonary disease in urban and rural China: a community-based survey of 25000 adults across 10 regions. BMJ Open Respir Res. 2018;5:e000267. doi:10.1136/bmjresp-2017-000267

23. Liu Y, Zhong L, Yuan S, et al. Why patients prefer high-level healthcare facilities: a qualitative study using focus groups in rural and urban China. BMJ Glob Health. 2018;3:e000854. doi:10.1136/ bmjgh-2018-000854

24. Fuhrrman C, Roche N, Vergne'gre A, et al. Hospital admissions related to acute exacerbations of chronic obstructive pulmonary disease in France, 1998-2007. Respir Med. 2011;105:595. doi:10.1016/j. rmed.2010.11.014

25. de Miguel-diez J, Jimenez-Garcia R, Hernandez-Barrera V, et al. Readmissions following an initial hospitalization by COPD exacerbation in Spain from 2006 to 2012. Respirology. 2016;21:489-496. doi: $10.1111 /$ resp. 12705

26. Stefan MS, Shieh M-S, Pekow PS, et al. Trends in mechanical ventilation among patients hospitalized with acute exacerbations of COPD in the United States, 2001 to 2011. Chest. 2015;147:959-968. doi: $10.1378 /$ chest. $14-1216$
27. Lindenauer PK, Stefan MS, Shieh M-S, et al. Outcomes associated with invasive and noninvasive ventilation among patients hospitalized with exacerbations of chronic obstructive pulmonary disease. JAMA Intern Med. 2014;174:1982-1993. doi:10.1001/ jamainternmed.2014.5430

28. Osadnik CR, Tee VS, Carson-Chahhoud KV, et al. Non-invasive ventilation for the management of acute hypercapnic respiratory failure due to exacerbation of chronic obstructive pulmonary disease. Cochrane Database Syst Rev. 2017;7:CD004104. doi:10.1002/ 14651858.CD003881.pub4

29. Chen W, FitzGerald JM, Sin DD, et al. Excess economic burden of comorbidities in COPD: a 15-year population-based study. Eur Respir J. 2017;50:pii: 1700393. doi:10.1183/13993003.003932017

30. Deniz S, Sengul A, Aydemir Y, et al. Clinical factors and comorbidities affecting the cost of hospital-treated COPD. Int J Chron Obstruct Pulmon Dis. 2016;11:3023-3030. doi:10.2147/COPD. $\mathrm{S} 120637$

31. Liu H, Wang N, Chen W, et al. Hospitalization trends in adult patients with COPD and other respiratory diseases in northeast China from 2005 to 2015. Biomed Res Int. 2018;2018:1060497.

\section{Publish your work in this journal}

The International Journal of COPD is an international, peer-reviewed journal of therapeutics and pharmacology focusing on concise rapid reporting of clinical studies and reviews in COPD. Special focus is given to the pathophysiological processes underlying the disease, intervention programs, patient focused education, and self management protocols. This journal is indexed on PubMed Central, MedLine and CAS. The manuscript management system is completely online and includes a very quick and fair peer-review system, which is all easy to use. Visit http://www.dovepress.com/testimonials.php to read real quotes from published authors. 